\title{
Slope Illuminance Prediction under a Cloudless Sky Based on the Novel Model for Global Horizontal Irradiance and Illuminance
}

\author{
Bo Liang ${ }^{1}$ and Wei $\mathrm{He}^{1,2, *}$ \\ ${ }^{I}$ School of Civil Engineering, Chongqing Jiaotong University, Chongqing 400074, China \\ ${ }^{2}$ Department of Traffic and Municipal Engineering, Sichuan College of Architectural Technology, Chengdu 610399, China
}

Received 3 June 2021; Accepted 12 September 2021

\begin{abstract}
Global illuminance on an inclined plane is affected by many factors, such as geographic longitude, latitude, time, weather, plane orientation, and inclination angle. To accurately and economically predict any slope illuminance under a cloudless sky, this study proposes a calculation model for the global horizontal irradiance and illuminance under a cloudless sky. The diffuse illuminance on the tilted surface was calculated by using the Perez point source model. The relationship between the global horizontal irradiance or illuminance and the solar altitude angle under a cloudless sky was established to address the reliance of the Perez point source model on real-time test data. The measured data from the French station of the International Daylighting Measurement Program was used to verify the global horizontal irradiance and illuminance and the global vertical north, east, south, and west illuminance. The global illuminance on an arbitrary inclined plane was verified using the measured data from China. Graphical and statistical methods were used to evaluate the error of the forecast data, and the reasons for the error were analyzed. Results show that the global horizontal irradiance and illuminance under a cloudless sky are linear functions of the solar altitude angle. The relative root mean square error (RRMSE) of the predicted global horizontal irradiance and illuminance are $5.4 \%$ and $10.2 \%$, respectively. The proposed calculation method of illuminance on a tilted surface does not rely on real-time measured data, significantly saving in test costs and predicting illuminance at any inclination angle and orientation under a cloudless sky. Except for the RRMSE of the global vertical north illuminance of $19.8 \%$, the RRMSE of the predicted global illuminance on the inclined plane in other orientations does not exceed $10.2 \%$. This study provides an essential reference for predicting luminance in the access zone of a road tunnel.
\end{abstract}

Keywords: Illuminance, Irradiance, Horizontal, Direct, Diffuse

\section{Introduction}

Highway construction has been accelerating day by day with the rapid economic development. In areas with complex terrain, especially mountainous areas, the number of road tunnels increases, inevitably resulting in a substantial increase in energy consumption. Therefore, the power consumption of tunnel lighting equipment must be reduced under the premise of ensuring traffic safety.

The luminance in the access zone of a tunnel $\left(L_{20}\right)$ is one of the benchmark parameters of tunnel lighting design, and the setting of this parameter has a significant impact on tunnel investment and operating electricity costs. The Tokyo Bay Subsea Tunnel in Japan is made the following comparisons in the design: under the same conditions, $L_{20}$ is set to $4000 \mathrm{~cd} / \mathrm{m}^{2}$ and $6000 \mathrm{~cd} / \mathrm{m}^{2}$, respectively, resulting in a $34 \%$ difference in equipment costs and a $30 \%$ difference in annual power consumption [1]. In recent years, the intelligent lighting of tunnels can adjust the tunnel lighting according to real-time $L_{20}$ [1] and realize energy-saving under the premise of ensuring driving safety.

The influencing factors of $L_{20}$ include the reflectance of the scenery outside the tunnel, the proportion of the scenery in the driver's field of view, and the illuminance

*E-mail address: hewei4912058@163.com

ISSN: 1791-2377 @ 2021 School of Science, IHU. All rights reserved.

doi:10.25103/jestr.144.17 outside the tunnel. Therefore, the illuminance outside the tunnel must be obtained to predict $L_{20} \cdot L_{20}$ is difficult to predict because the outdoor illuminance varies with the geographical longitude and latitude, the time, the weather, and other factors. The most accurate way to obtain illuminance is to establish a long-term measurement station as the International Daylighting Measurement Program (IDMP) does. However, the operating cost of the measurement station is high, and installing illuminance meters on all surfaces with diverse orientations and tilt angles to collect the required data is impractical. Therefore, estimating the global illuminance on the inclined plane ( $I L_{\beta g}$ ) has practical significance. If the measured value is unavailable, then modeling is the best alternative to estimating the illuminance.

On this basis, scholars have conducted considerable work on estimating $I L_{\beta g}$ through a mathematical model [2, 3]. Nevertheless, problems in predictability, operability, and cost remain. Therefore, accurately and economically predicting $I L_{\beta g}$ under a cloudless sky is a pressing issue.

To this end, this study simplified the influence of aerosols on irradiance and illuminance. Global horizontal irradiance $\left(I R_{h g}\right)$ and illuminance $\left(I L_{h g}\right)$ models were developed to predict $I L_{\beta g}$ under a cloudless sky without relying on real-time test data as input parameters, providing 
a reference for predicting $L_{20}$.

\section{State of the art}

Many studies focused on the field of irradiance [4-6]. Illuminance is the visible light part of irradiance, and the calculation method of irradiance on an inclined surface is also applicable to illuminance. $I L_{\beta g}$ includes the solar direct, sky diffused, and ground reflection components. The models of the solar direct and ground reflection components in the existing literature are the same, and the main difference is in the calculation of the diffused component on an inclined plane. The diffused illuminance on an inclined plane $\left(I L_{\beta d}\right)$ could usually be calculated by the sky distribution or inclined plane models [7]. In the sky distribution model, $I L_{\beta d}$ is obtained by the double integral of the luminance of the sky element $[3,8]$. The luminance of the sky element could use the formula recommended by the International Commission on Illumination or could be measured using a charge-coupled device camera [9] or a sky scanner. However, problems of the sky distribution model are in determining the sky classification [10-12] and solving the integral. The inclined plane models are divided into isotropic and anisotropic models. The $I L_{\beta d}$ of both is calculated according to the horizontal diffused illuminance ( $\left.I L_{h d}\right)$. Hay, Klucher, and Perez are three well-known anisotropic models. The isotropic model assumes that the entire sky is uniform. Hay's model [13] assumes that the sky is composed of the circumsolar region and a uniform sky. Klucher's model [14] assumes that overcasts are isotropic, while clear days show strong horizon brightening. The sky hemisphere of the Perez model [15] is divided into three regions. That is, two anisotropic regions are superimposed on the isotropic background: the circumsolar brightening due to the forward scattering of aerosols and the horizon brightening due to multiple Rayleigh scattering and backscattering under the clear atmosphere. The Perez model performs the best among the three anisotropic models [16, 17]. Li et al. [18] found that the Perez model performs better with local coefficients than with those proposed by Perez. Robledo et al. [19, 20] proposed that the accuracy of the Perez model would be significantly improved when the coefficients are determined independently for each orientation. Robledo and Soler [21] developed a simple and more accurate model for when the surface does not "see" the circumsolar disk. The premise of the inclined plane model could obtain $I L_{h d}$. $I L_{h d}$ could be obtained using the irradiance-illuminance conversion models, machine intelligence, and empirical formula. Multiplying the horizontal diffused irradiance $\left(I R_{h d}\right)$ by the luminous efficiency could obtain $I L_{h d}$ in the irradianceilluminance conversion model [22]. At present, many weather stations worldwide can provide horizontal global, horizontal diffused, and normal direct irradiance but not outdoor illuminance. The input parameters of the horizontal diffused luminous efficacy proposed by Perez et al. [2] include the atmospheric precipitable content, the zenith angle, the sky's brightness, and the coefficients determined by the sky's clearness. Therefore, the irradiance-illuminance conversion model requires $I R_{h d}$, the normal incidence direct irradiance $\left(I R_{i s}\right)$, and the dew point temperature provided by the weather station as input parameters. The conversion model could not predict $I L_{h d}$ in advance. Data sets could be used to establish a model of the input value and $I L_{h d}$ through machine intelligence. The developed model is more accurate than the regression model but lacking in terms of physical explanation [23-25]. To use machine intelligence, a measure station must be established locally to obtain sufficient training data [26, 27]. However, the cost of establishing and operating a measure station is high. Darula et al. [28] summarized the typical ratios of $I L_{h d}$ and extraterrestrial horizontal illuminance under 15 sky types. However, the error of the predicted $I L_{h d}$ at a specific moment with this typical ratio is significant in most cases. Petrzala et al. [29] proposed a clear sky physical model for calculating solar irradiance and illuminance. The model considers the actual atmospheric pollution conditions and the optical parameters of atmospheric aerosols. Meanwhile, a software solution was developed. Wandji et al. [30] introduced a method for estimating the global illuminance and its normal incidence direct component under cloud-free conditions. The input of this method includes the total column content of ozone and water vapor and the aerosol optical properties generated by the Copernicus Atmosphere Monitoring Service.

The aforementioned studies has shortcomings in predicting $I L_{\beta g}$, but studies that can accurately predict $I L_{\beta g}$ under a cloudless sky at low cost are rare. In this study, the Perez point source model was used to calculate $I L_{\beta d}$. To address the reliance of the Perez point source model on realtime test data, a model for $I R_{h g}$ and $I L_{h g}$ under a cloudless sky is proposed. The measured data of the cloudless sky at the French IDMP station are used to verify $I R_{h g}, I L_{h g}$, and the global vertical north, east, south, and west illuminance. $I L_{\beta g}$ is verified using the measured data under a cloudless sky from China. Moreover, an error analysis of the forecast data is carried out using graphics and statistical methods.

The remainder of the study is organized as follows. The third section discusses the construction of a method of predicting $I L_{\beta g}$ under a cloudless sky. The fourth section verifies the predicted illuminance of the cloudless sky through the data set and the measured data. Furthermore, the reasons for the error are analyzed. The last section summarizes the study and presents relevant conclusions.

\section{Methodology}

\subsection{Prediction of illuminance on a tilted surface}

The illuminance on a tilted surface is calculated as the sum of the sky-diffuse, direct beam, and ground-reflected components and expressed as follows:

$$
I L_{\beta g}=I L_{\beta d}+I L_{\beta s}+I L_{\beta r}
$$

where $I L_{\beta \mathrm{g}}, I L_{\beta d}, I L_{\beta s}$, and $I L_{\beta r}$ are the global illuminance, diffuse illuminance, direct illuminance, and reflected illuminance on the tilted surface (lux).

The input parameters of the direct illuminance on the tilted surface include the normal incidence direct illuminance $I L_{i s}$ (lux) and the incidence angle of sunlight on an inclined surface $\theta_{\beta i}$ (degrees). 
$I L_{\beta s}=I L_{i s} \cos \theta_{\beta i}$

The classic calculation formula for normal incidence direct illuminance is as follows [28]:

$$
I L_{i s}=I L_{e x} \cdot E_{c} \cdot \exp \left(-m \cdot a_{v} \cdot T_{v}\right)
$$

where $I L_{\mathrm{ex}}$ is the constant of the extraterrestrial illuminance perpendicular to the sunbeams (133800 lux), $E_{\mathrm{c}}$ is the ellipticity correction representing the daily change in the distance between the earth and the sun (dimensionless), $m$ is the air mass (dimensionless), $\mathrm{a}_{v}$ is the luminous extinction under a dry and clean atmosphere (dimensionless), and $T_{v}$ is the illuminance turbidity factor (dimensionless). According to the approximate expected value of the illuminance turbidity factor on sunny days recommended by Darula and Kittler [31], the value of $T_{v}$ is 2.5 for a cloudless sky.

The $E_{\mathrm{c}}$ recommended by Kittler is used [32].

$E_{c}=1+0.0337 \cos \left[\left(N_{1}-4^{\circ}\right) 360 / 365\right]$

where $N_{1}$ is the day number of the year (dimensionless). For example, January 2, 2021 is the 2nd day of the year 2021, so $N_{1}=2$.

$$
m=\frac{1}{\sin \gamma_{s}+0.50572\left(\gamma_{s}+6.07995\right)^{-1.6364}}
$$

where $\gamma_{s}$ is the solar altitude angle (degrees).

$$
a_{v}=\frac{1}{10.1+0.045 m}
$$

The incident angle of sunlight on the inclined plane is obtained using Formula (7):

$$
\cos \theta_{\beta i}=\cos \gamma_{s} \sin \beta \cos (\psi-\Theta)+\sin \gamma_{s} \cos \beta
$$

where $\beta$ is the surface inclination (degrees), $\psi$ is the azimuth angle that the surface faces (radians), and $\Theta$ is the azimuth of the sun (radians).

The reflected and diffused components have fewer intensity changes at different times of the year than the direct component, making them a very reliable light source in daylight projects. The ground reflected illuminance on the tilted surface is determined by global horizontal illuminance $I L_{h g}$ (lux), ground reflectivity $R_{g}$ (dimensionless), and the surface inclination (Formula (8)).

$$
I L_{\beta r}=R_{g} I L_{h g} 0.5(1-\cos \beta)
$$

Ineichen et al. [33] proposed that ground reflectivity distinguishes north, east, south, and west and morning and afternoon. Eight different reflectances were formed, which were the average of the three-month measurement period. To simplify the prediction, this study uses a single ground reflectivity. The value is the average of the above eight reflectances, which is 0.15 .

The diffused illuminance on the tilted surface is estimated using the Perez point source model [2], which is expressed as follows:

$I L_{\beta d}=I L_{h d}\left[0.5(1+\cos \beta)\left(1-F_{1}\right)+F_{1}\left(a_{1} / a_{2}\right)+F_{2} \sin \beta\right](9)$

where $I L_{h d}$ is the diffuse horizontal illuminance (lux), $F_{1}$ is the circumsolar brightening coefficient (dimensionless), and $F_{2}$ is the horizon brightening coefficient (dimensionless). $a_{1}$ and $a_{2}$ are expressed as follows:

$$
\begin{aligned}
& a_{1}=\max \left(0, \cos \theta_{\beta i}\right) \\
& a_{2}=\max \left(\cos 85^{\circ}, \cos Z\right)
\end{aligned}
$$

where $Z$ is the solar zenith angle (radians). $Z, F_{1}$, and $F_{2}$ are expressed as follows:

$$
\begin{aligned}
& Z=\frac{\pi}{180}\left(90-\gamma_{s}\right) \\
& F_{1}=f_{11}+f_{12} \Delta+f_{13} Z \\
& F_{2}=f_{21}+f_{22} \Delta+f_{23} Z
\end{aligned}
$$

where $f_{11}, f_{12}, f_{13}, f_{21}, f_{22}$, and $f_{23}$ are the Perez model coefficients for illuminance (dimensionless), as shown in Table 1; $\Delta$ is the sky's brightness (dimensionless), which represents the optical transparency of the clouds. $\Delta$ is expressed as

$$
\Delta=m \frac{I R_{h d}}{I R_{\mathrm{e} x}}
$$

where $I R_{h d}$ is the diffuse horizontal irradiance $\left(\mathrm{W} / \mathrm{m}^{2}\right)$, and $I R_{\mathrm{e} x}$ is the constant of the extraterrestrial irradiance $\left(1366 \mathrm{~W} / \mathrm{m}^{2}\right)$.

Table 1. Perez model coefficients for illuminance

\begin{tabular}{l|l|l|l|l|l|l|l}
\hline$\varepsilon$ & $\begin{array}{l}\text { Upper } \\
\text { limit }\end{array}$ & $f_{11}$ & $f_{12}$ & $f_{13}$ & $f_{21}$ & $f_{22}$ & $f_{23}$ \\
\hline 1 & 1.065 & 0.011 & 0.57 & -0.081 & -0.095 & 0.158 & -0.018 \\
2 & 1.23 & 0.429 & 0.363 & -0.307 & 0.05 & 0.008 & -0.065 \\
3 & 1.5 & 0.809 & -0.054 & -0.442 & 0.181 & -0.17 & -0.092 \\
4 & 1.95 & 1.014 & -0.252 & -0.531 & 0.275 & -0.35 & -0.096 \\
5 & 2.8 & 1.282 & -0.42 & -0.689 & 0.38 & -0.56 & -0.114 \\
6 & 4.5 & 1.426 & -0.653 & -0.779 & 0.425 & -0.79 & -0.097 \\
7 & 6.2 & 1.485 & -1.214 & -0.784 & 0.411 & -0.63 & -0.082 \\
8 & - & 1.17 & -0.3 & -0.615 & 0.518 & -1.89 & -0.055 \\
\hline
\end{tabular}

$\varepsilon$ is the sky's clearness (dimensionless) in Table 1 . The change in $\varepsilon$ reflects the changing atmospheric turbidity and cloud cover. $\varepsilon$ is expressed as follows:

$$
\varepsilon=\frac{\frac{I R_{i s}+I R_{h d}}{I R_{h d}}+1.041 Z^{3}}{1+1.041 Z^{3}}
$$


where $I R_{i s}$ is the normal incidence direct irradiance $\left(\mathrm{W} / \mathrm{m}^{2}\right)$. The $I R_{i s}$ under cloudless sky conditions is expressed as [34]

$$
I R_{i s}=1353 * 0.7^{m^{0.678}}
$$

\subsection{Prediction of the solar altitude and azimuth}

Solar altitude and azimuth are essential parameters for predicting the global illuminance on an inclined plane. They are functions of geographic location and time. The solar altitude angle is calculated using Formula (18) [35].

$\gamma_{s}=\frac{180}{\pi} \arcsin (\sin \omega \sin \delta+\cos \omega \cos \delta \cos A)$

where $\pi$ is the constant (radians), $\omega$ is the geographic latitude (degrees), $\delta$ is the solar declination angle (degrees), and $A$ is the solar hour angle (degrees).

Solar declination is expressed as follows:

$$
\begin{aligned}
& \delta=0.3723+23.2567 \sin \zeta+0.1149 \sin 2 \zeta-0.1712 \sin 3 \zeta \\
& -0.758 \cos \zeta+0.3656 \cos 2 \zeta+0.0201 \cos 3 \zeta
\end{aligned}
$$

$\zeta$ (radians) is calculated using Formula (20):

$\zeta=\frac{2 \pi\left(N_{1}-N_{0}\right)}{365.2422}$

$N_{0}=0.2422(Y-1985)+79.6764-\mathrm{INT}\left(\frac{Y-1985}{4}\right)$

where $Y$ is the year. INT is a function for obtaining an integer.

Solar time is denoted as $t_{s}(\mathrm{~h})$. The solar hour angle is a linear function of solar time.

$A=15\left(t_{s}-12\right)$

$t_{s}=\mathrm{t} \pm \frac{15\left|z_{t}\right|-L_{l o c}}{15}+$

$\frac{0.0028-1.9857 \sin \zeta+9.9059 \sin 2 \zeta-7.0924 \cos \zeta-0.6882 \cos 2 \zeta}{60}$

where $t$ is the local standard time $(\mathrm{h}), z_{t}$ is the time zone (dimensionless), and $L_{l o c}$ is the local longitude (degrees). The "+" sign applies to the Western Hemisphere, and the "-" sign applies to the Eastern Hemisphere.

The expressions of $\Theta$ in the morning $\left(t_{s} \leq 12\right)$ and afternoon $\left(t_{s}>12\right)$ are different (Formulas (24) and (25)) [35]. $\Theta$ is less than $\pi$ radians in the morning and greater than $\pi$ radians in the afternoon.

$$
\begin{aligned}
& t_{s} \leq 12, \Theta=\pi-\arccos \frac{\sin \gamma_{s} \sin \omega-\sin \delta}{\cos \gamma_{s} \cos \omega} \\
& t_{s}>12, \Theta=\pi+\arccos \frac{\sin \gamma_{s} \sin \omega-\sin \delta}{\cos \gamma_{s} \cos \omega}
\end{aligned}
$$

3.3 Proposed models for global horizontal illuminance and irradiance

$I R_{h d}$ and $I L_{h d}$ are required to calculate $I L_{\beta g} . I R_{h d}$ and $I L_{h d}$ can be obtained from the IDMP station. Although shadow band correction factors are used, the measurement errors of $I R_{h d}$ and $I L_{h d}$ are still significant. In addition, many areas have no IDMP stations. $I R_{h d}$ and $I L_{h d}$ can be obtained by the following equations:

$I L_{h d}=I L_{h g}-I L_{i s} \sin \gamma_{s}$

$I R_{h d}=I R_{h g}-I R_{i s} \sin \gamma_{s}$

where $I R_{h g}$ is the global horizontal irradiance $\left(\mathrm{W} / \mathrm{m}^{2}\right)$.

$I R_{h g}$ can be calculated according to clear sky models $[36,37]$. However, the use of clear sky models is low because collecting atmospheric input parameters and implementing these models are difficult tasks. The clear sky models cannot predict $I R_{h g}$ because the models rely on realtime measurement data.

Under a cloudless sky, the extraterrestrial irradiance is assumed to reach the ground when the solar altitude angle is 90 degrees. That is, the reduced irradiance reaching the ground due to the absorption or scattering by aerosols is negligible at this time. Given that the $I R_{h g}$ under a cloudless sky is linearly related to $\gamma_{s}$, the $I R_{h g}$ under a cloudless sky is expressed as follows:

$I R_{h g}=\frac{I R_{\mathrm{e} x}}{90} \gamma_{s}=15.178 \gamma_{s}$

Similarly, the $I L_{h g}$ under a cloudless sky is expressed as

$I L_{h g}=\frac{I L_{e x}}{90} \gamma_{s}=1486.667 \gamma_{s}$

The verification of Equations (28) and (29) is presented in Section 4. The 29 formulas above can be programmed in Excel to improve forecasting efficiency. The input parameters after programming are shown in Table 2. All input parameters can be easily obtained.

Table 2. Input parameters for predicting the global illuminance on the tilted plane

\begin{tabular}{c|l|l|l}
\hline Date & $t$ & $z_{t}$ & Eastern or Western Hemisphere \\
\hline$L_{l o c}$ & $\omega$ & $\beta$ & $\psi$ \\
\hline
\end{tabular}

\section{Result Analysis and Discussion}

\section{1 data source}

The measurement data from the IDMP station is used to verify the prediction of the illuminance on the inclined surface. The IDMP stations are of two types. Illuminance and irradiance are the primary measurement parameters at the general type IDMP station; sky luminance distribution and other meteorological quantities are recorded at the research type IDMP station. This study cites data from the IDMP station located in France. The basic situation of the station is shown in Table 3. The global vertical north, east, 
south, and west illuminance are denoted as $I L_{n v g}, I L_{e v g}, I L_{s v g}$, and $I L_{w v g}$ respectively. The data provided by the station include the test date, $t, \gamma_{s}, \Theta, I L_{h g}, I L_{h d}$, $I L_{n v g}, I L_{e v g}, I L_{s v g}, I L_{w v g}, I R_{h g}, I R_{h d}, I R_{h s}$, and the shadow band correction factors. The collection equipment for all illuminance is LMT BAP 30 FCT. The test equipment for $I R_{h g}$ and $I R_{h d}$ is Kipp \& Zonen CM21 and Kipp \& Zonen CM6, respectively. Kipp \& Zonen CH-1 is used to measure the $I R_{i s}$ on the EKO STR-22 solar tracker. All equipment are calibrated regularly. In addition, all data passed the ENTPE quality control program.

Table. 3. basic situation of the station

\begin{tabular}{l|l}
\hline Site & $\begin{array}{l}\text { France, Vaulx-en-Velin, located in the eastern } \\
\text { part of the Lyon urban community }\end{array}$ \\
\hline Code of IDMP & FRA2 \\
Categories of IDMP & $\begin{array}{l}\text { General type } \\
45.7786 \mathrm{~N}\end{array}$ \\
Latitude & $4.9225 \mathrm{E}$ \\
Longitude & $170 \mathrm{~m}$ \\
Altitude & $\begin{array}{l}\text { GMT+1 } \\
\text { The climate is moderate and influenced by the } \\
\text { ocean (Mediterranean). The average sunshine } \\
\text { hour is 2,100 hours. The mean number of foggy } \\
\text { days is 55 days, mainly in winter. } \\
\text { Climate } \\
\text { characteristics }\end{array}$ \\
$\begin{array}{l}\text { Data type } \\
\text { Initial measurements }\end{array}$ \\
$\begin{array}{l}\text { Since September 1991 } \\
\text { ENTPE, Rue Maurice Audin, 69518 Vaulx-en- } \\
\text { Velin, FRANCE. dumortier@entpe.fr, } \\
\text { Phone:(33) 472047087, http://idmp.entpe.fr/. }\end{array}$ \\
\hline
\end{tabular}

This study selects data from the data set according to the following four principles:

1) Although more data are available, only the data from 2018 to 2020 are selected. The data of these three years are believed capable of reflecting the law of long-term observation.

2) The data without clouds during the entire day are chosen. Although the data set provides 1-min instantaneous illuminance and irradiance, the station only provides approximate sky types every $2 \mathrm{~h}$. The illuminance and the irradiance that show an excellent regular change over time are selected to obtain data without clouds during the entire day.

3) Although the test equipment is installed on the roof, when $\gamma_{s}$ is small, the sunlight is blocked by the surrounding buildings. The measured illuminance and irradiance are the values after the sunlight is blocked. The illuminance and irradiance prediction formulas in this study are all based on the premise of no shading. For better verification, the $\gamma_{s}$ corresponding to the selected data is greater than 15 degrees.

4) To reduce the amount of data processing, this study only uses the data every 10 minutes in the data set. For example, data at 9:00, 9:10, and 9:20 is selected.

After strict screening, a total of 12 cloudless skies in Vaulx-en-Velin France from 2018 to 2020 meet the above four conditions. The scatterplot of $t$ versus the measured $I R_{h g}, I L_{h g}, I L_{n v g}, I L_{s v g}, I L_{e v g}$, and $I L_{w v g}$ for 12 cloudless skies at Vaulx-en-Velin France are shown in Figs. 1-6. Fig. 3 indicates that $I L_{n v g}$ is smaller than the other orientations, and the fluctuation range is also smaller because the vertical surface facing north does not receive sunlight. The illuminance in other orientations fluctuates wildly. The surface facing south can always receive sunlight during the day. Figs. 5and 6 show that surfaces facing east and west only receive sunlight in the morning and afternoon, respectively. Figs. 1, 2, and 4 show that the maximum $I R_{h g}$, $I L_{h g}$, and $I L_{s v g}$ occur at noon. Figs. 5 and 6 show that the maximum $I L_{\text {evg }}$ and $I L_{\text {wvg }}$ occur at around 9:00 and 17:00, respectively.

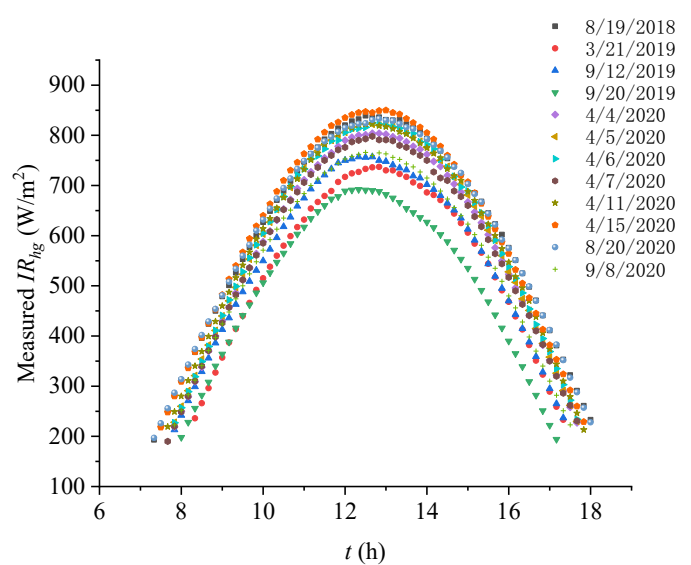

Fig. 1. Scatterplot of $t$ vs. the measured $I R_{h g}$ for 12 cloudless skies at Vaulx-en-Velin France

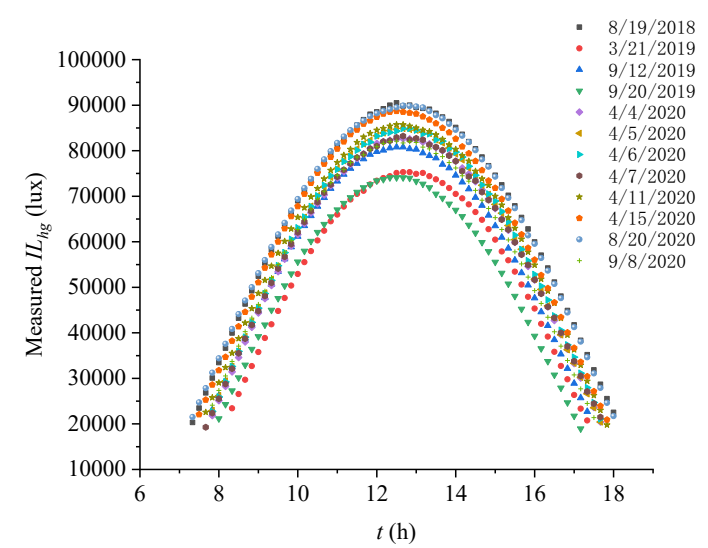

Fig. 2. Scatterplot of $t$ vs. the measured $I L_{h g}$ for 12 cloudless skies at Vaulx-en-Velin France

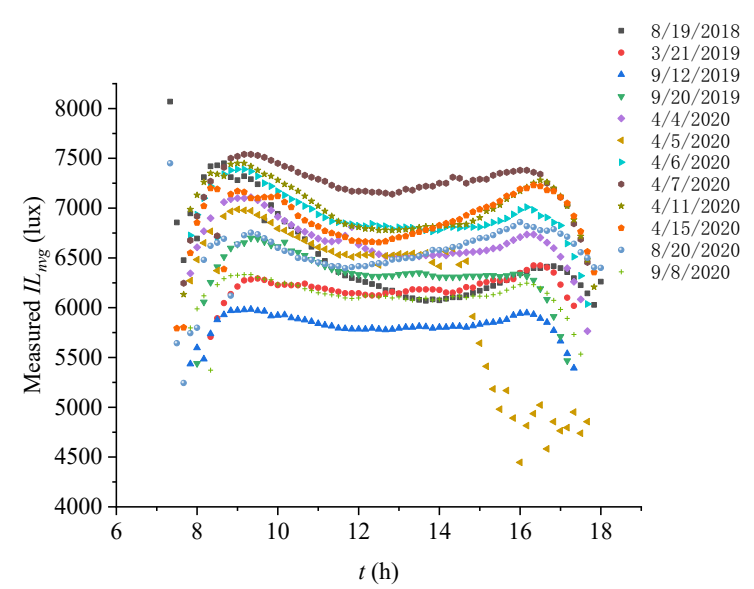

Fig. 3. Scatterplot of $t$ vs. the measured $I L_{n v g}$ for 12 cloudless skies at Vaulx-en-Velin France 


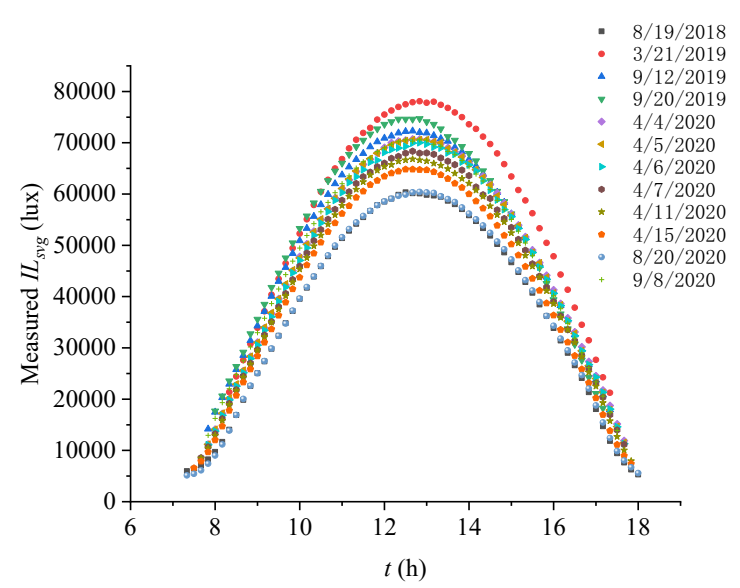

Fig. 4. Scatterplot of $t$ vs. the measured $I L_{\text {svg }}$ for 12 cloudless skies at Vaulx-en-Velin France

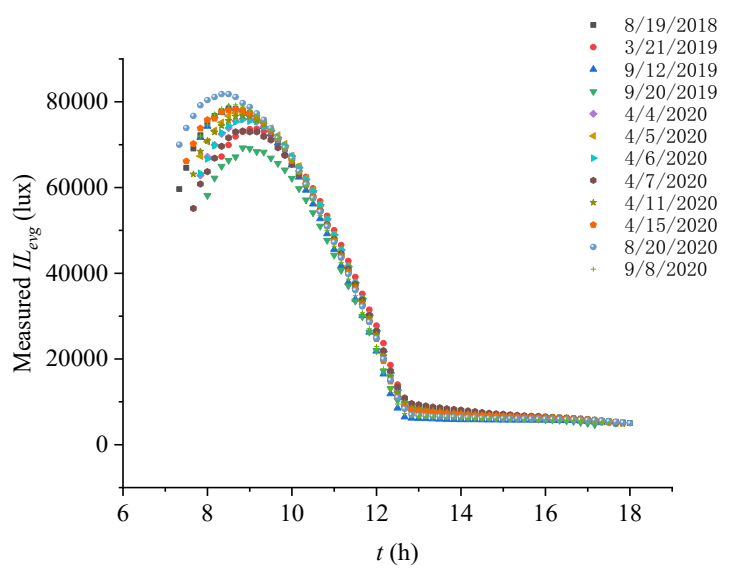

Fig. 5. Scatterplot of $t$ vs. the measured $I L_{\text {evg }}$ for 12 cloudless skies at Vaulx-en-Velin France

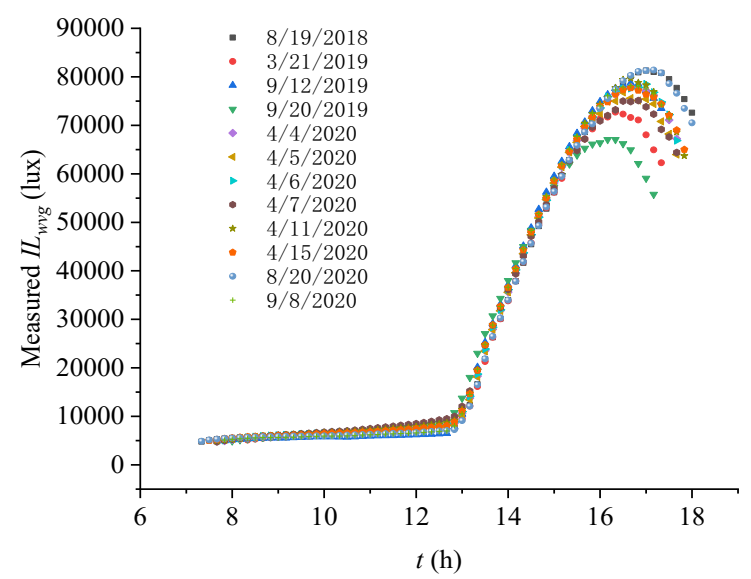

Fig. 6. Scatterplot of $t$ vs. the measured $I L_{\text {wrg }}$ for 12 cloudless skies at Vaulx-en-Velin France

\subsection{Verification of global horizontal irradiance and illuminance}

The measured $I R_{h g}$ or $I L_{h g}$ is linearly related to $\gamma_{s}$ as shown in Figs. 7 and 8 . The measured data verify Formulas (28) and (29). Figs. 9 and 10 respectively show the comparison results between the measured and predicted values of $I R_{h g}$ and $I L_{h g}$. The predicted $I R_{h g}$ is more consistent with the measured value than $I L_{h g}$.

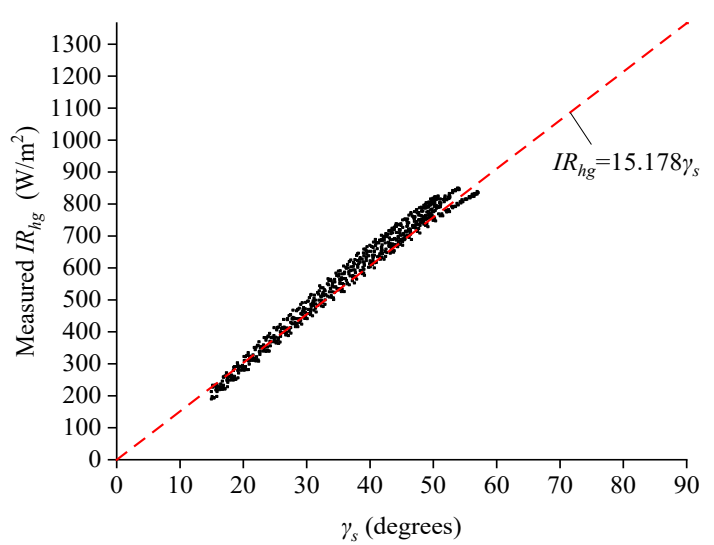

Fig. 7. Scatterplot of $\gamma_{s}$ vs. the measured $I R_{h g}$

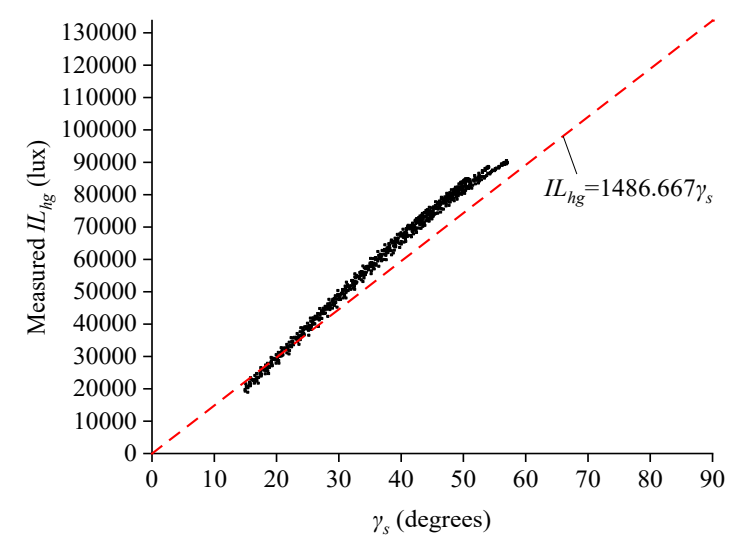

Fig. 8. Scatterplot of $\gamma_{s}$ vs. the measured $I L_{h g}$

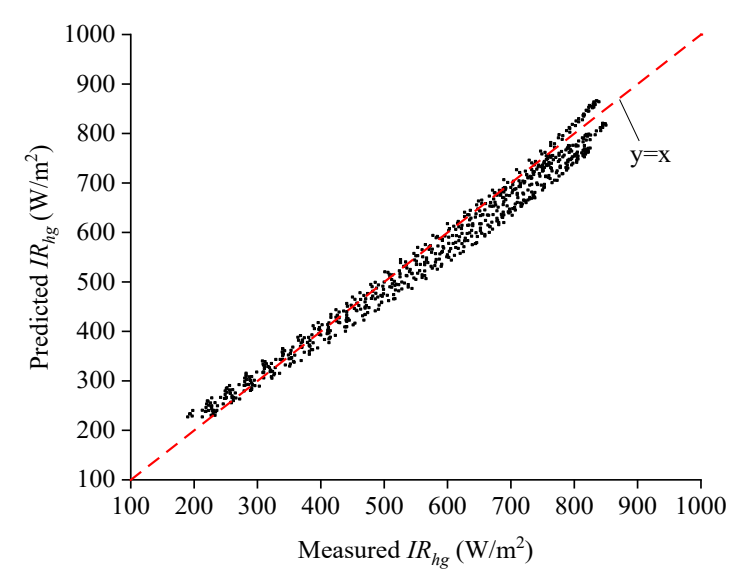

Fig. 9. Scatterplot of the measured $I R_{h g}$ vs. the predicted $I R_{h g}$

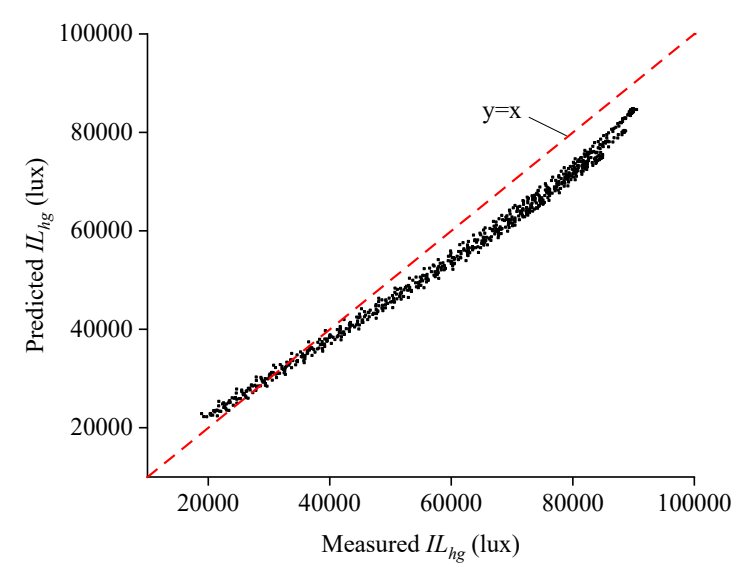

Fig. 10. Scatterplot of the measured $I L_{h g}$ vs. the predicted $I L_{h g}$ 
The histograms shown in Figs. 11 and 12 depict the frequency distribution of the relative error. The relative errors of $I R_{h g}$ and $I L_{h g}$ are concentrated at $-10 \%-10 \%$ and $20 \%-10 \%$, respectively.

relative error $=\frac{100(P-M)}{M}$

where $P$ and $M$ are the predicted and measured values, respectively

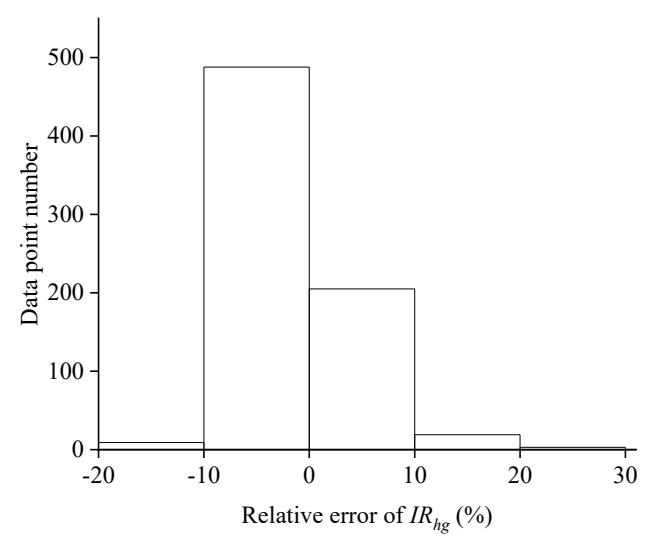

Fig. 11. Relative error histogram of $I R_{h g}$

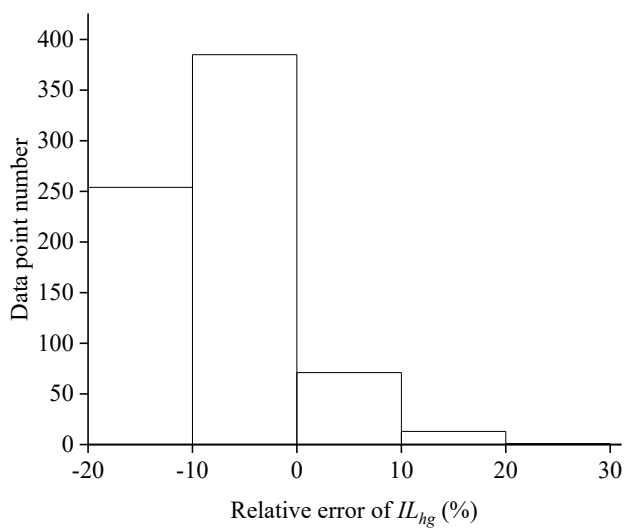

Fig. 12. Relative error histogram of $I L_{h g}$

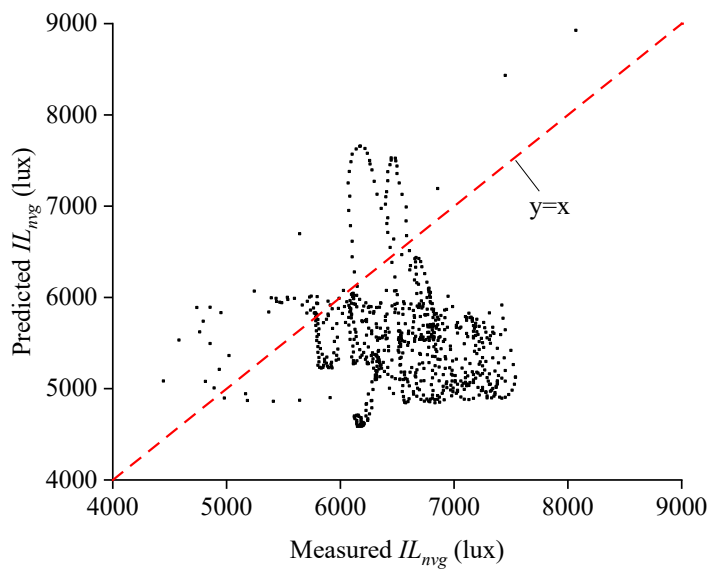

Fig. 13. Scatterplot of the measured $I L_{n v g}$ vs. the predicted $I L_{n v g}$

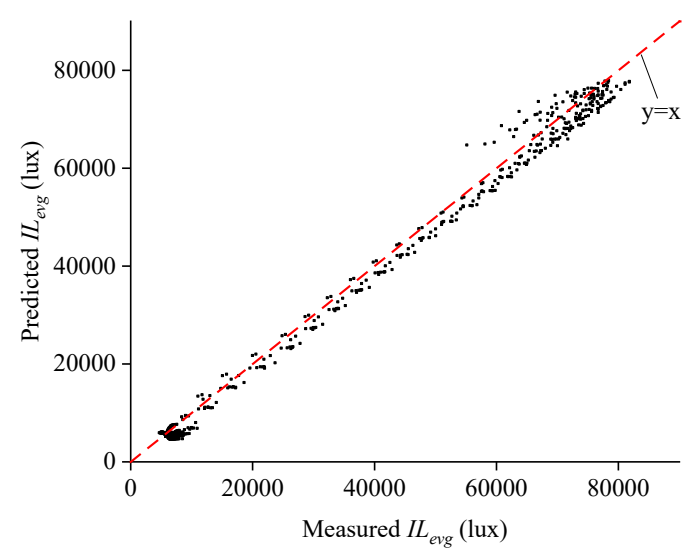

Fig. 14. Scatterplot of the measured $I L_{e v g}$ vs. the predicted $I L_{e v g}$

\subsection{Verification of global vertical illuminance}

The verification results of the global vertical illuminance are shown in Figs. 13-16. The predicted $I L_{n v g}$ is quite different from the measured value, and the predicted illuminance in the other three orientations is near the measured value. The relative error frequency distribution of the global vertical illuminance is shown in Figs. 17-20. The relative errors of $I L_{n v g}$ and $I L_{\text {evg }}$ are concentrated in $-30 \%-0$ and $-10 \%-0$, respectively; the relative errors of $I L_{s v g}$ and $I L_{w v g}$ are mostly $-10 \%-10 \%$.

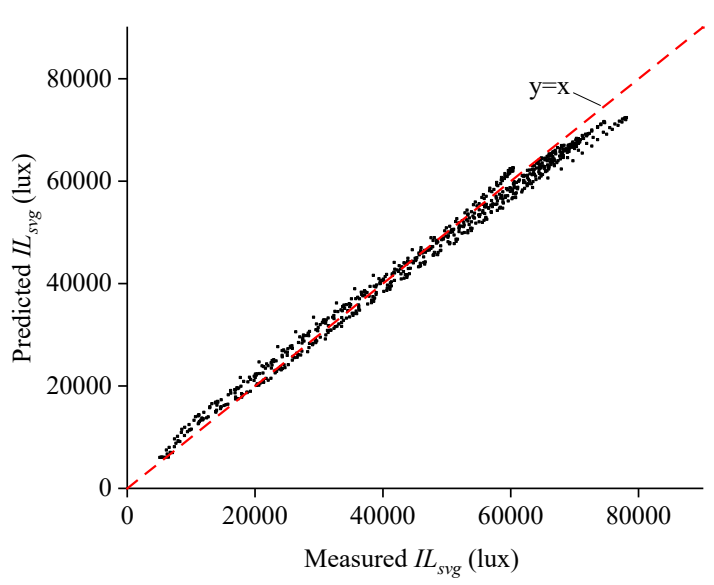

Fig. 15. Scatterplot of the measured $I L_{s v g}$ vs. the predicted $I L_{s v g}$

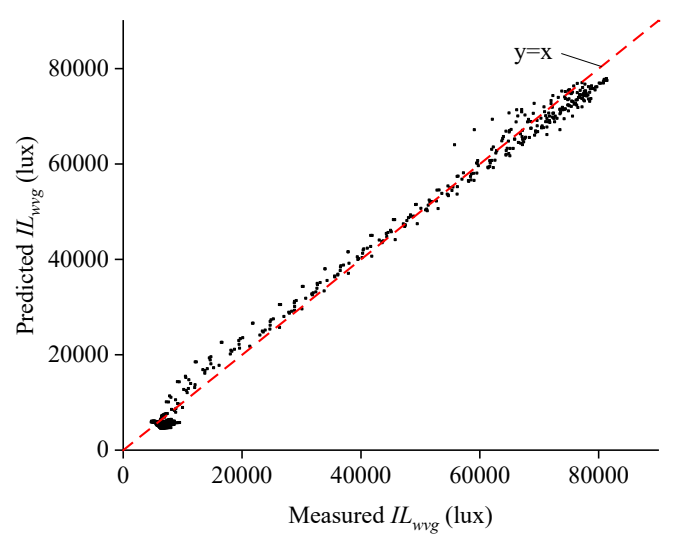

Fig. 16. Scatterplot of the measured $I L_{w v g}$ vs. the predicted $I L_{w v g}$ 


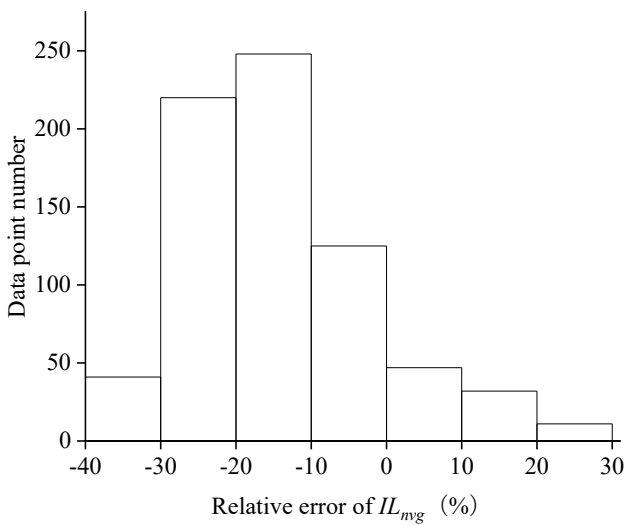

Fig. 17. Relative error histogram of $I L_{n v g}$

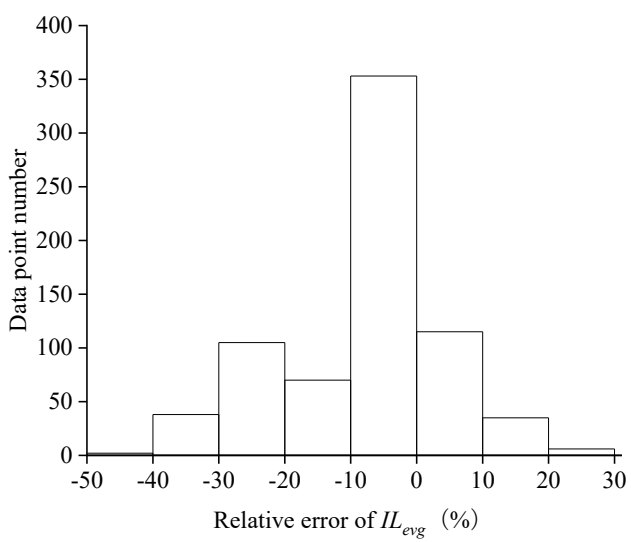

Fig. 18. Relative error histogram of $I L_{e v g}$

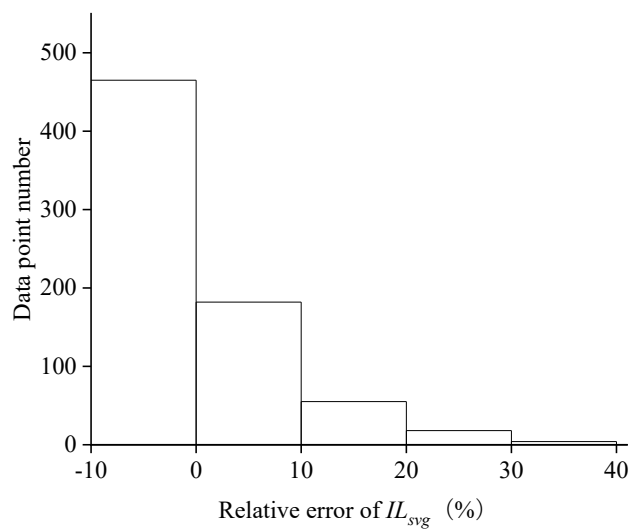

Fig. 19. Relative error histogram of $I L_{s v g}$

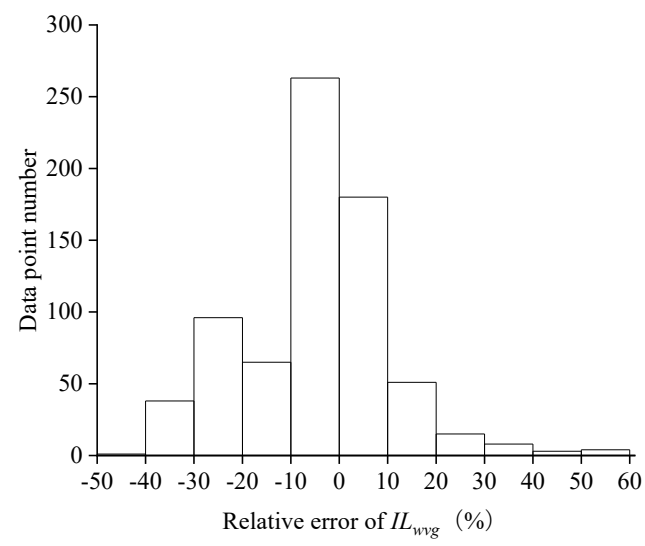

Fig. 20. Relative error histogram of $I L_{w v g}$
The relative mean bias error (RMBE) and the relative root mean square error (RRMSE) are employed to obtain the forecast data's overall error. Table 4 shows the RMBE and RRMSE of the six forecast data.

$\mathrm{RMBE}=\frac{100 \sum_{1}^{n}(P-M)}{n \cdot \overline{\mathrm{M}}}$

where $n$ is the data point number, and $\bar{M}$ is the average of the measured values.

RRMSE $=\frac{100 \sqrt{\frac{\sum_{1}^{n}(P-M)^{2}}{n}}}{\bar{M}}$

Table. 4. Statistical results of the overall error of the six forecast data

\begin{tabular}{l|l|l|l|l|l|l}
\hline & $I R_{h g}$ & $I L_{h g}$ & $I L_{\text {nvg }}$ & $I L_{\text {evg }}$ & $I L_{\text {svg }}$ & $I L_{\text {wvg }}$ \\
\hline RMBE (\%) & -2.8 & -8.5 & -14.9 & -4.1 & -1.6 & -1.7 \\
RRMSE (\%) & 5.4 & 10.2 & 19.8 & 7.5 & 4.7 & 7.0 \\
\hline
\end{tabular}

The overall errors of illuminance in different directions shown in Table 4 are sorted as follows:

$I L_{n v g}>I L_{h g}>I L_{e v g}>I L_{w v g}>I L_{s v g}$

The prediction of $I R_{h g}$ is more accurate than that of $I L_{h g}$. The error of the predicted $I L_{n v g}$ is obvious. The predicted $I L_{s v g}$ shows reasonably good agreement with the measured data, indicating that the anisotropic diffused component on the sunlight surface is well estimated. This conclusion is the same as that of Li et al. [16].

4.4 Verification of global illuminance on an arbitrary tilted surface

Given that the French IDMP station only provides $I L_{n v g}, I L_{s v g}, I L_{\text {evg }}$, and $I L_{w v g}$, the verification of the global illuminance on an arbitrary inclined plane is based on the measured data in Deyang, Sichuan Province, China. The selected test site has a mild climate and an open terrain. No obstacles can cause measurement errors. Moreover, the reflection characteristics are the same for all orientations. The color of the vegetation does not change significantly throughout the year, so the ground reflectivity does not change over time. The test site is located at 104.416667 degrees east longitude and 31.0905556 degrees north latitude. The time zone is GMT +08 , and the surface inclination is 29 degrees. The data under the cloudless skies are shown in Table 5. The uncertainty of the illuminance meter (TES-1339R, TES Electrical Electronic Corp, Taipei, Taiwan, China) is $\pm 3 \%$ reading \pm 5 digits. The equipment was calibrated before use.

Table 5. Data under cloudless skies

\begin{tabular}{l|c|c|c|c}
\hline Date & $t$ & $\psi$ & Predicted & Measured \\
& & (degrees) & $I L_{\beta \mathrm{g}}(\mathbf{l u x})$ & $I L_{\beta \mathrm{g}}$ (lux) \\
\hline $2 / 5 / 2021$ & $12: 50$ & 101 & 66921 & 69130 \\
$2 / 5 / 2021$ & $13: 01$ & 96 & 62394 & 63850 \\
$2 / 5 / 2021$ & $13: 21$ & 192 & 89161 & 93440 \\
$2 / 5 / 2021$ & $13: 33$ & 180 & 89410 & 94530 \\
$2 / 5 / 2021$ & $13: 35$ & 250 & 70744 & 77220 \\
$2 / 5 / 2021$ & $13: 54$ & 10 & 21242 & 30270
\end{tabular}




\begin{tabular}{c|c|c|c|c}
$2 / 5 / 2021$ & $13: 56$ & 84 & 43948 & 48380 \\
$2 / 5 / 2021$ & $13: 58$ & 170 & 85920 & 92490 \\
$2 / 5 / 2021$ & $13: 59$ & 254 & 71679 & 77690 \\
$2 / 5 / 2021$ & $15: 20$ & 17 & 11422 & 21550 \\
$2 / 5 / 2021$ & $15: 21$ & 86 & 21458 & 27800 \\
$2 / 5 / 2021$ & $15: 22$ & 163 & 65962 & 63550 \\
$2 / 5 / 2021$ & $15: 24$ & 260 & 71335 & 69400 \\
$5 / 29 / 2020$ & $8: 59$ & 32 & 68946 & 69560 \\
$5 / 29 / 2020$ & $9: 02$ & 121 & 76612 & 79320 \\
$5 / 29 / 2020$ & $9: 24$ & 215 & 34264 & 39790 \\
$5 / 29 / 2020$ & $9: 55$ & 302 & 36658 & 43350 \\
$5 / 29 / 2020$ & $10: 24$ & 20 & 78496 & 82360 \\
$5 / 29 / 2020$ & $10: 56$ & 111 & 104609 & 112400 \\
$5 / 29 / 2020$ & $11: 24$ & 193 & 90449 & 96450 \\
$5 / 29 / 2020$ & $11: 54$ & 294 & 80548 & 78330 \\
$5 / 29 / 2020$ & $13: 04$ & 13 & 98026 & 95520 \\
$5 / 29 / 2020$ & $13: 38$ & 102 & 96705 & 99360 \\
$5 / 29 / 2020$ & $14: 06$ & 193 & 107784 & 116500 \\
$5 / 29 / 2020$ & $14: 36$ & 285 & 105744 & 111200 \\
$5 / 29 / 2020$ & $15: 08$ & 45 & 58895 & 53980 \\
$5 / 29 / 2020$ & $15: 42$ & 137 & 51715 & 47760 \\
\hline
\end{tabular}

The comparison between the measured $I L_{\beta g}$ and the predicted $I L_{\beta g}$ is shown in Fig. 21. Overall, the measured $I L_{\beta g}$ is near the predicted $I L_{\beta g}$. The relative error of $I L_{\beta g}$ shown in Fig. 22 is mostly $-10 \%-10 \%$. Table 5 shows that the relative error is significant when the measured $I L_{\beta g}$ is small. Table 6 exhibits the overall error assessment of $I L_{\beta g}$.

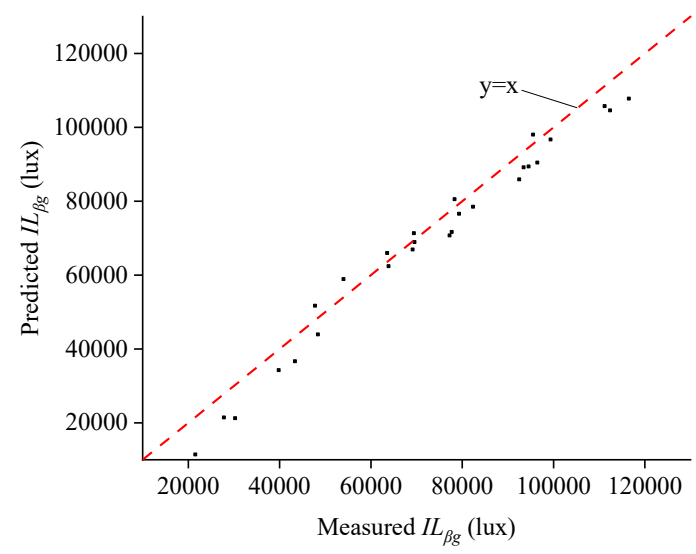

Fig. 21. Scatterplot of the measured $I L_{\beta g}$ vs. the predicted $I L_{\beta g}$

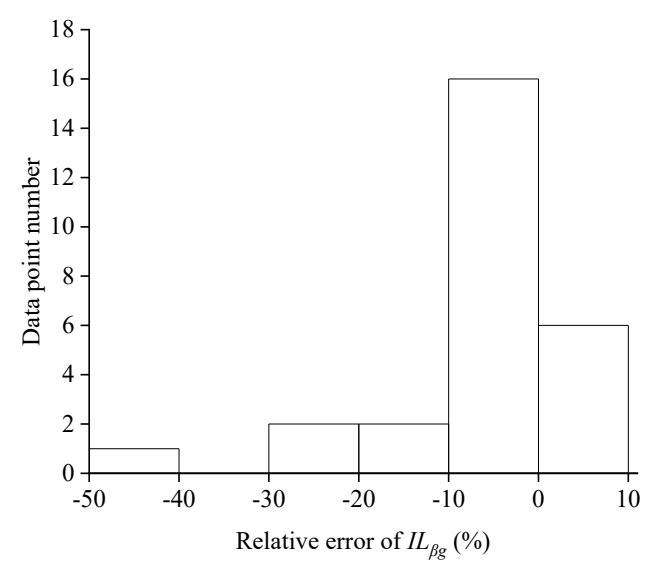

Fig. 22. Relative error histogram of $I L_{\beta g}$

Table 6. Overall error assessment of $I L_{\beta g}$

\begin{tabular}{l|l}
\hline & $I L_{\beta g}$ \\
\hline RMBE (\%) & -4.8 \\
\hline RRMSE (\%) & 7.4 \\
\hline
\end{tabular}

\subsection{Error Analysis}

Among the three components of $I L_{\beta g}, I L_{\beta d}$ is the largest potential source of prediction error. In most cases, the prediction error of $I L_{\beta r}$ is less than the error of $I L_{\beta d}$. The prediction error of $I L_{\beta s}$ is the smallest of the three [17]. Although the best Perez model is used to predict $I L_{\beta d}$, among all the illuminance verifications, the relative error of $I L_{n v g}$ is the largest when the data from the French station is used because $I L_{n v g}$ is equal to its diffused component in most cases. The verification from China shows that the relative error of $I L_{\beta g}$ is also apparent when $I L_{\beta g}$ is small because $I L_{\beta d}$ contributes the most to $I L_{\beta g}$.

The $I R_{h g}$ and $I L_{h g}$ models proposed in this study assume that the extraterrestrial irradiance and illuminance all reach the ground when the solar altitude angle is 90 degrees under a cloudless sky. This assumption is impossible because the absorption or scatter of the aerosol reduces the amount reaching the ground. Therefore, the prediction of $I R_{h g}$ and $I L_{h g}$ with the proposed models has errors, leading to an error in the predicted $I L_{\beta g}$.

Another source of error is the values of $T_{v}$ and $R_{g}$. In this study, the value of $T_{v}$ is 2.5 to make predictions independent of real-time aerosol parameter values. Nevertheless, under a cloudless sky, the number of solid particles and the water vapor content in the air can affect the value of $T_{v}$ [31]. Different orientations and times affect the value of $R_{g}$ [33]. The value of $R_{g}$ is 0.15 in this study to facilitate forecasting.

The sky conditions are not recorded every minute. The French station only provides an approximate sky type every $2 \mathrm{~h}$. Although only the illuminance and irradiance that show an excellent regular change over time are selected, determining which data used corresponds to a cloudless sky is impossible, which can cause severe deviations in some of the verification results.

The measurement errors can affect the input value and propagate to the resulting output. The ideal situation with a perfect input value cannot be achieved. Although the selected data passed the quality control procedures, inherent errors in the input value can lead to prediction bias.

\section{Conclusions}

To predict $I L_{\beta g}$ under a cloudless sky, this study proposes a novel model for $I R_{h g}$ and $I L_{h g}$. Combining theoretical research with existing data sets and measured data, the predicted values of $I R_{h g}, I L_{h g}, I L_{n v g}, I L_{s v g}, I L_{\text {evg }}, I L_{w v g}$, and $I L_{\beta g}$ were verified. Then, the errors were analyzed. Finally, the following conclusions were obtained.

(1) Under a cloudless sky, the proposed $I R_{h g}$ and $I L_{h g}$ are linear functions of $\gamma_{s}$. The overall error of the proposed model is small. Moreover, the prediction of $I R_{h g}$ is more accurate than that of $I L_{h g}$.

(2) The proposed calculation method for $I L_{\beta g}$ under a cloudless sky does not rely on real-time test data. The 
method can significantly save in test costs.

(3) The proposed method system can predict illuminance at any inclination angle and orientation under a cloudless sky. Except for the RRMSE of $I L_{n v g}$, which is $19.8 \%$, the RRMSE values of the other $I L_{\beta g}$ do not exceed $10.2 \%$.

This study proposes a method for predicting $I L_{\beta g}$ under a cloudless sky. The established model is simplified, highly maneuverable, and nearly zero-cost. The proposed method has important reference significance for the study of $L_{20}$. Given the lack of measured data sets for the polar, cold, arid, and equatorial climate zones, this study only verified the predicted illuminance in the temperate climate zone. In future studies, the predicted illuminance using the proposed method will be compared with the measured illuminance for the polar, cold, arid, and equatorial climate zones to provide a more comprehensive understanding of the influence of climate zones on $I L_{\beta g}$.

\section{Acknowledgements}

This study was supported by the National Natural Science Foundation of China (Grant No. 51878107).

This is an Open Access article distributed under the terms of the Creative Commons Attribution License.

\section{References}

1. He, W.,Liang, B., "A Novel Method for Calculating Luminance in the Access Zone of a Road Tunnel based on a Digital Camera". Journal of Engineering Science and Technology Review, 14(3), 2021, pp.148 - 157.

2. Perez, R., Ineichen, P., Seals, R., Michalsky, J.,Stewart, R., "Modeling daylight availability and irradiance components from direct and global irradiance". Solar Energy, 44(5), 1990, pp.271289.

3. Li, D. H., Lau, C. C.,Lam, J. C., "Predicting daylight illuminance on inclined surfaces using sky luminance data". Energy, 30(9), 2005, pp.1649-1665.

4. Choosri, P., Foobunma, K.,Kongsomlit, A., "A Method to Estimation of Global Solar Radiation with Meteorological Parameters under Cloudless Sky Condition using Artificial Neural Network". Naresuan University Journal: Science and Technology, 29(4), 2021, pp.1-12.

5. Ruiz-Arias, J. A., "Aerosol transmittance for clear-sky solar irradiance models: Review and validation of an accurate universal parameterization". Renewable and Sustainable Energy Reviews, 145(1), 2021, pp.111061.

6. Srivastava, S.,Lessmann, S., "A comparative study of LSTM neural networks in forecasting day-ahead global horizontal irradiance with satellite data". Solar Energy, 162(1), 2018, pp.232-247.

7. Li, D. H.,Lou, S., "Review of solar irradiance and daylight illuminance modeling and sky classification”. Renewable Energy, 126(1), 2018, pp.445-453.

8. Torres, J. L.,García, I., "Analytical expressions for estimating sky diffuse irradiance and illuminance on tilted planes for the CIE Standard General Skies". Renewable Energy, 174(1), 2021, pp.320335.

9. Yun, S.-I.,Kim, K.-S., "Sky luminance measurements using CCD Camera and comparisons with Calculation models for predicting indoor illuminance”. Sustainability, 10(5), 2018, pp.1556.

10. Alshaibani, K.,Li, D., "Sky type classification for the ISO/CIE Standard General Skies: a proposal for a new approach". International Journal of Low-Carbon Technologies, 1(1), 2021, pp. 1 .

11. Alshaibani, K. A., "Classification Standard Skies: The use of horizontal sky illuminance”. Renewable and Sustainable Energy Reviews, 73(1), 2017, pp.387-392.

12. Lou, S., Li, D. H.,Lam, J. C., "CIE Standard Sky classification by accessible climatic indices”. Renewable Energy, 113(1), 2017, pp.347-356.

13. Hay, J. E.,Davies, J. A., "Calculation of the solar radiation incident on an inclined surface ". In: Proc. 1st Canadian Solar Radiation Data Workshop (Edited by J.E. Hay and T.K. Won), Toronto, Canada: Supply and Services Canada, 1980, pp.59-72.

14. Klucher, T. M., "Evaluation of models to predict insolation on tilted surfaces". Solar Energy, 23(2), 1979, pp.111-114.

15. Perez, R., Stewart, R., Arbogast, C., Seals, R.,Scott, J., "An anisotropic hourly diffuse radiation model for sloping surfaces: description, performance validation, site dependency evaluation". Solar Energy, 36(6), 1986, pp.481-497.

16. Li, D. H.,Lam, J. C., "Evaluation of slope irradiance and illuminance models against measured Hong Kong data". Building and Environment, 35(6), 2000, pp.501-509.
17. Perez, R., Seals, R., Ineichen, P., Stewart, R.,Menicucci, D., “A new simplified version of the Perez diffuse irradiance model for tilted surfaces". Solar Energy, 39(3), 1987, pp.221-231.

18. Li, D.,Lam, J., "Evaluation of Perez slope irradiance and illuminance models against measured Hong Kong data". International journal of ambient energy, 20(4), 1999, pp.193-204.

19. Robledo, L.,Soler, A., "Point-source Perez illuminance model: Dependence of coefficients on surface orientation at Madrid". International Journal of Lighting Research and Technology, 28(3), 1996, pp.141-148.

20. Robledo, L.,Soler, A., "Dependence on surface orientation of coefficients in the circumsolar simplified Perez illuminance model for vertical planes at Madrid". Energy Conversion and Management, 39(15), 1998, pp.1585-1595.

21. Robledo, L.,SOLER, A., "Modelling irradiance on inclined planes with an anisotropic model". Energy, 23(3), 1998, pp.193-201.

22. Littlefair, P. J., "Measurements of the luminous efficacy of daylight". Lighting Research \& Technology, 20(4), 1988, pp.177188.

23. Kaytez, F., Taplamacioglu, M. C., Cam, E.,Hardalac, F., "Forecasting electricity consumption: A comparison of regression analysis, neural networks and least squares support vector machines". International Journal of Electrical Power \& Energy Systems, 67(1), 2015, pp.431-438.

24. Zou, L., Wang, L., Lin, A., Zhu, H., Peng, Y.,Zhao, Z., "Estimation of global solar radiation using an artificial neural network based on an interpolation technique in southeast China". Journal of Atmospheric Solar-Terrestrial Physics, 146(1), 2016, pp.110-122.

25. Dong, N., Chang, J.-F., Wu, A.-G.,Gao, Z.-K., "A novel convolutional neural network framework based solar irradiance prediction method". International Journal of Electrical Power Energy Systems, 114(1), 2020, pp.105411.

26. Belaid, S.,Mellit, A., "Prediction of daily and mean monthly global solar radiation using support vector machine in an arid climate". Energy Conversion and Management, 118(1), 2016, pp.105-118.

27. Qing, X.,Niu, Y., "Hourly day-ahead solar irradiance prediction using weather forecasts by LSTM". Energy, 148(1), 2018, pp.461468.

28. Darula, S., Kittler, R.,Wittkopf, S. K., "Outdoor illuminance levels in the tropics and their representation in virtual sky domes". Architectural Science Review, 49(3), 2006, pp.301-313.

29. Petržala, J., Kómar, L.,Kocifaj, M., "An advanced clear-sky model for more accurate irradiance and illuminance predictions for arbitrarily oriented inclined surfaces". Renewable Energy, 106(1), 2017, pp.212-221.

30. Wandji Nyamsi, W., Blanc, P., Dumortier, D., Mouangue, R., Arola, A.,Wald, L., "Using Copernicus Atmosphere Monitoring Service (CAMS) Products to Assess Illuminances at Ground Level under Cloudless Conditions". Atmosphere, 12(5), 2021, pp.643.

31. Darula, S.,Kittler, R., "New trends in daylight theory based on the new ISO/CIE Sky Standard: 3. Zenith luminance formula verified by measurement data under cloudless skies". Building Research Journal, 53(1), 2005, pp.9-31.

32. Kittler, R.,Darula , S., "Determination of sky types from global illuminance". International Journal of Lighting Research and Technology, 32(4), 2000, pp.187-193. 
33. Ineichen, P., Perez, R.,Seals, R., "The importance of correct albedo determination for adequately modeling energy received by tilted surfaces”. Solar Energy, 39(4), 1987, pp.301-305.

34. Meinel, A.,Meinel, M., "Applied Solar Energy ". Boston: Addison Wesley Publishing Co., America, 1976, pp.37-43.

35. Wu, Y., Liu, C.,Wen, S., "Calculation of Sky Luminance Distribution at Arbitrary Time Based on the CIE Sky Model". Acta Optiaa Sinica, 34(11), 2014, pp.27-33.
36. Sun, X., Bright, J. M., Gueymard, C. A., Acord, B., Wang, P.,Engerer, N. A., "Worldwide performance assessment of 75 global clear-sky irradiance models using principal component analysis". Renewable Sustainable Energy Reviews, 111(1), 2019, pp.550-570.

37. Yang, D., "Choice of clear-sky model in solar forecasting". Journal of Renewable and Sustainable Energy, 12(2), 2020, pp.026101. 